\title{
Creencias del amor romántico en adolescentes: una intervención desde la investigación-acción
}

\section{Beliefs of romantic love in adolescents: an action research intervention}

\section{Yeni Alejandra HeRnández Domínguez* \\ ANA DE LOURDES CASTRO RÍOS** \\ ERIKA EGLEONTINA BARRIOS GONZÁLEZ ${ }^{* * *}$}

El propósito de esta investigación fue modificar las creencias del amor romántico de un grupo de adolescentes para prevenir la violencia en las relaciones amorosas. El artículo hace visible cómo, a través del amor romántico, se justifica y tolera la violencia en las relaciones de pareja. Se trata de una intervención participativa con un grupo de adolescentes para reflexionar sobre la díada amor romántico y violencia. La metodología fue cualitativa y se utilizó el diseño investigación-acción, compuesto por cuatro fases: planificación, acción, observación y reflexión. Los resultados indican que trabajar desde un enfoque participativo permite a los adolescentes generar cambios significativos de las percepciones, creencias y significados que tenían sobre el amor de pareja, el noviazgo y la violencia. La conclusión revela que los adolescentes son capaces de modificar sus creencias a partir de intervenciones que favorezcan una reflexión informada.

The purpose of this investigation was to modify the romantic love beliefs of a group of adolescents as a way to prevent violence in romantic relationships. The article is characterized by making visible how, through romanticized love, violence in relationships is tolerated and justified. Hence, an approach to the subject is made through a participatory intervention with a group of adolescents to reflect on the dyad romantic love and violence. The methodology was qualitative, and the research-action design was used, made up offour phases: planning, action, observation and reflection. The results indicate that working from a participatory approach allowed adolescents to generate significant changes in the perceptions, beliefs, and meanings they had about love from a partner, courtship, and violence. It is concluded that adolescents are able to modify their beliefs through interventions that allow them informed reflection.

\section{Palabras clave:} creencias, amor romántico, violencia, adolescentes, investigaciónacción

\section{Keywords:}

beliefs, romantic love, violence, adolescents, action research

Recibido: 17 de febrero de 2020 | Aceptado para su publicación: 24 de agosto de 2020| Publicado: 26 de agosto de 2020

Recuperado de: https://sinectica.iteso.mx/index.php/SINECTICA/article/view/1095 doi: 10.31391/S2007-7033(2020)0055-007

\footnotetext{
* Maestra en Ciencias en Intervención Psicosocial por la Universidad Juárez Autónoma de Tabasco. Miembro del Semillero de Investigadores. Líneas de investigación: psicología y creencias del amor romántico entre adolescentes. Correo electrónico: yeni_1125997@hotmail.com/https://orcid.org/0000-0003-2416-7211

** Doctora en Estudio de las Sociedades Latinoamericanas, Mención Sociología, por la Universidad ARCIS-Chile. Académica de la Escuela de Trabajo Social, perteneciente a la Facultad de Ciencias Sociales y Económicas de la Universidad Católica del Maule, Chile. Líneas de investigación: familias, familias rurales, resiliencia, jóvenes, género, mediación familiar e investigación cualitativa. Correo electrónico: acastro@ucm.cl/https://orcid.org/0000-0002-2840-6921

*** Doctoranda en Psicología de la Universidad Autónoma del Estado de Morelos. Docente y coordinadora del Semillero de Investigadores, un proyecto orientado a la promoción de la investigación de esta universidad. Líneas de investigación: competencias en investigación, dificultades de aprendizaje, desarrollo humano y educación superior. Correo electrónico: erika.barriosgon@uaem.edu.mx/ https://orcid.org/0000-0001-6214-0779
} 


\section{INTRODUCCIón}

\section{El amor romántico como normalizador de la violencia de pareja}

El amor romántico o de pareja es sumamente importante en la vida de las personas; sin embargo, el estereotipo y las características adjudicadas a ese sentimiento dejan al descubierto que la violencia es disfrazada en nombre del amor.

De acuerdo con la Encuesta Nacional de Dinámica de las Relaciones en los Hogares (INEGI, 2016), en México, de los 46.5 millones de adolescentes de quince años y más que residen en el país, 30.7 millones de ellas (66.1\%) han padecido al menos un incidente de violencia en sus relaciones sentimentales. Las estadísticas revelan que el $43.9 \%$ de las mujeres que tienen o tuvieron un noviazgo han experimentado violencia en algún momento de su relación. Cuando la violencia de pareja no se identifica ni aborda de manera oportuna en sus primeras etapas puede terminar en feminicidios. Según datos de la Organización Mundial de la Salud (OMS, 2017), el 38\% de los asesinatos de mujeres que se producen en el mundo son cometidos por su pareja masculina.

Así pues, pareciera que estar en una relación amorosa representa un terreno fértil para experimentar conductas de control, abusos y chantajes frecuentemente. En ese sentido, el amor romántico juega un papel esencial en el establecimiento y la normalización de la violencia en un contexto de pareja (Ferrer y Bosch, 2013).

En nuestra sociedad se mantiene vigente un modelo de amor romántico asociado a creencias que idealizan a la pareja y desestiman sus defectos y comportamientos negativos; se sobrevalora el sacrificio y el sufrimiento por amor. Estos aspectos favorecen y perpetúan la violencia en las relaciones de pareja (Marroquí y Cervera, 2014). Ferrer y Bosch (2013) consideran que "las características del amor romántico y las altas expectativas que genera (inalcanzables en la mayoría de los casos) pueden provocar importantes consecuencias personales (insatisfacción, frustración, sufrimiento)" (p. 23). Estos elementos contribuyen a una idea distorsionada y tóxica del amor.

Desde la perspectiva de Yela (2002), las creencias falsas sobre el amor romántico que permanecen vigentes en la sociedad son interiorizadas y descritas como la verdadera naturaleza del amor. Al respecto, Ferrer y Bosch (2013) señalan los riesgos de interiorizar algunas creencias, como la del amor todo lo puede; esta lleva a pensar que se puede vencer cualquier problema y que es posible cambiar a la pareja (así muestre comportamientos agresivos). Esta creencia posterga la posibilidad de dejar una relación violenta, y de seguir creyendo que los celos son un sinónimo de amor o considerarlos como muestras de mayor interés y amor. Esto justifica los chantajes, el control y la posesión, lo que limita la libertad. Creer que el amor de pareja da sentido a sus vidas predispone a que las personas se aferren a mantenerse en la relación pese a que experimenten problemas.

De esa manera, conductas que debieran categorizarse como violentas son encubiertas como supuestas manifestaciones de amor o cariño hacia la pareja (Galicia, Sánchez y Robles, 2013; Bonilla-Algovia y Rivas-Rivero, 2018). En ese sentido, diversos autores han confirmado que existe una simbiosis entre el constructo de amor romántico y la violencia (Borrajo, Gámez \& Calvete, 2015; Caro y Monreal, 2017; Rodríguez-Castro, Lameiras Fernández y Carrera, 2015). Por ende, es necesario la coeducación en jóvenes que contribuya a una reformulación del concepto e ideas 
erróneas del amor que ayude a establecer relaciones sobre la base del respecto, la confianza y el compromiso (Bosch, Ferrer y Alzamora, 2006).

\section{Aportes de la perspectiva de género en la comprensión del amor romántico}

El amor es un constructo multidimensional a partir del cual se prescriben un sinnúmero de características; una de ellas gira en torno a las representaciones que se configuran a través del género; los roles y estereotipos de género disponen las cualidades que deben tener hombres y mujeres al momento de emparejarse (Rodríguez, 2017).

Los aportes de la perspectiva de género permiten comprender de qué manera nos construimos socialmente como hombres o como mujeres, y se moldean nuestras concepciones acerca de lo que es el amor o, más en específico, el amor romántico, que se ha convertido en la piedra angular desde la cual se puede explicar por qué una pareja se mantiene unida a pesar de la presencia de violencia explícita e implícita, y se desestiman el impacto y los alcances que esta tiene en su vida.

Para Lamas (citado en Guzmán y Bolio, 2010), la perspectiva de género es un mecanismo que ayuda a reelaborar los conceptos de hombre y mujer, en virtud de que aspectos como la sexualidad, el matrimonio y el amor se ven radicalmente afectados. Las representaciones románticas son sustentadas por "las representaciones culturales, entre las que sobresalen [...] los roles de género” (Rodríguez, 2017, p. 24). Mediante esta lógica, se decodifica una serie de manifestaciones, representaciones e imaginarios que entran en juego en las relaciones amorosas. Por ende, es imprescindible retomar la mirada de la perspectiva de género para comprender el papel de la construcción de este en las concepciones sobre el amor.

De acuerdo con Bosch, Ferrer y Gili (1999), en cada sociedad se producen diferencias y desigualdades en función del sexo, del cual emanan la interiorización de significados que no dependen de lo biológico, sino de aspectos culturales y sociales. Desde esta perspectiva, las construcciones sociales instruyen las formas de expresión, los significados y las experiencias que presentan una introyección diferente en hombres y mujeres.

\section{Construcciones del amor a la luz de la socialización diferenciada de género}

Nos regimos por una estructura social que posiciona de modo desigual a hombres y mujeres, lo cual repercute decisivamente en el comportamiento, conductas, sentimientos, formas de entablar relaciones, así como los significados que adjudican al tema del amor (Esteban y Távora, 2008). Al respecto, Lagarde (2001) menciona que "mujeres y hombres aman, y lo hacen de maneras diferentes, con la creencia en la universalidad del amor y en que el amor es para unos y otros, la vía privilegiado a la felicidad" (p. 10). Sin embargo, esta idea no se traduce en igualdad dentro de las relaciones.

Así, el género, como construcción sociocultural, confluye para que hombres y mujeres desde la niñez sean moldeados y educados, y den respuesta a estereotipos y roles de género que se establecen socialmente. Valenzuela (2004) considera que es a través de los recursos de socialización temprana que se incorporan pautas de 
configuración psíquica y social que hacen posible la femineidad y la masculinidad. Mediante dicho proceso, las personas aprenden y hacen suyas las pautas de comportamiento que la sociedad impone a hombres y mujeres. Se ha constatado que hombres y mujeres reciben mensajes distintos como resultado de la socialización diferencial de género (Bosch et al., 1999).

Para comprender cómo las personas construyen e interiorizan el significado del amor romántico, es preciso reconocer la existencia de una socialización diferencial, la cual comienza en el nacimiento, puesto que desde la infancia se considera que niños y niñas son diferentes; por tanto, se les instruye para distintos papeles de la vida adulta (García, Hernández y Monter, 2019).

De esa manera, hombres y mujeres reciben mensajes diferentes que refuerzan el deber ser a través de roles y estereotipos de género, los cuales son admitidos con total naturalidad a consecuencia del proceso de socialización diferencial, que radica en la congruencia de los mensajes emitidos por todos los agentes socializadores, que están constituidos por la familia, la iglesia, la escuela y los medios de comunicación, ya que estos son parte del contexto inmediato donde se desenvuelven (Bosch et al., 1999).

Estos agentes de socialización reproducen y rectifican una y otra vez la posición que deben adoptar hombres y mujeres en cada momento y circunstancia de su vida. De ahí que en el momento en que se establecen relaciones de pareja se conforman y reproducen relaciones desiguales, en la medida en que hombres y mujeres son estructurados y estructuradores de la desigualdad y diferencia social, por estar sujetos a una condición de género de la que no se pueden divorciar de los imaginarios patriarcales (Valenzuela, 2004).

Desde este escenario se han reproducido las desigualdades mediante roles diferenciados que aparecen a la luz de las relaciones amorosas. Hombres y mujeres reciben mensajes distintos y ello se transpola a las relaciones de pareja, en las que una vez más entra en juego el aprendizaje adquirido durante el proceso de socializaciión y genera expectativas diversas en las formas de entender el amor.

El proceso de socialización diferencial atraviesa la vida de hombres y mujeres; de esta manera, configura la subjetividad de cada uno. Para Flores (2019), el concepto de amor romántico como construcción social se asume con mayor hincapié en las mujeres; como parte de la subjetividad femenina, se considera que la vida de las mujeres gira en torno al amor de pareja; internalizan la idea del príncipe azul que las rescatará e idealizan el amor de pareja; creen que son dependientes y se entregan a la búsqueda de protección y afecto. Estas características incluyen como ejes principales el tema del matrimonio y la maternidad.

De acuerdo con Lagarde (2001), el amor en la vida de las mujeres se vive como un deber. Esta autora menciona que "para las mujeres el amor es definitorio de su identidad de género" (p. 12). Culturalmente, ellas aprenden a amar, se les instruye para eso, de manera que es el objetivo principal de sus vidas, antes que ellas mismas y sus necesidades. Mientras que "los hombres, como sujetos amorosos, han sido construidos de tal forma que para ellos el amor se acaba, tiene un fin" (Lagarde, 2001, p. 73). La identidad masculina respecto al amor establece límites, por lo cual dicho sentimiento no constituye el principal interés en la vida de los hombres. 
Los patrones socioculturales se encuentran arraigados a la subjetividad, de tal modo que mantienen vigencia con el tiempo. De ahí que diversos autores han confirmado que existe una brecha de género cuando se aborda el tema del amor romántico (Caro, 2008; Caro y Monreal, 2017; Larrañaga, Yubero y Yubero, 2012; Cubells y Calsamiglia, 2015).

\section{Metodología}

Este trabajo tiene un enfoque cualitativo; utilizamos el diseño investigación-acción participativa, de acuerdo con la definición de Balcázar (2003): "La investigaciónacción involucra a los miembros de una comunidad o grupo en el proceso de investigación en una forma no tradicional, como agentes de cambio y no como objetos de estudio" (p. 60). Para la ejecución de la investigación-acción, retomamos el modelo de Latorre (2003), que establece cuatro fases: planificación, acción, observación y reflexión, así como las recomendaciones de Pedroza (2011).

\section{Participantes}

Los participantes fueron 13 adolescentes (siete hombres y seis mujeres), estudiantes de tercer grado de telesecundaria. Su edad promedio era de quince años. Fue un muestreo de tipo voluntario, porque invitamos a la comunidad académica a participar en el estudio. Solo tomamos para el análisis los datos de los estudiantes que concluyeron las cuatro fases del estudio.

\section{Contexto de la investigación}

En Tabasco, de acuerdo con la Encuesta Nacional de Dinámica de las Relaciones (INEGI, 2016), la prevalencia de violencia de pareja en mujeres entre quince años y más es del 40.1\%. Según el grado de severidad de la violencia de pareja, representa el $43.9 \%$, y se ubica por arriba de la media nacional.

El diagnóstico de violencia en el noviazgo en jóvenes de quince a dieciocho años llevado a cabo por el Instituto Estatal de las Mujeres (IEM-Tabasco, 2018) indica que el $69.8 \%$ de los adolescentes han experimentado actos de celos en su relación de noviazgo. Un $66.2 \%$ de mujeres y $81 \%$ de los hombres reconocen haber sido manipulados por su novio/a para tener relaciones sexuales y accedieron por miedo o temor. El $28.5 \%$ ha experimentado conducta de control, por ejemplo, que su pareja revise su celular.

Además de identificar los episodios de violencia que han vivido los jóvenes, el IEM-Tabasco presentó un panorama de las representaciones sociales que ocultan la violencia en las relaciones amorosas. En este sentido, el 51\% interioriza la creencia de que el amor todo lo puede, pues se siente optimista al pensar que su pareja sí puede modificar su comportamiento agresivo. El $14.6 \%$ cree que los celos son sinónimo de amor, y se considera culpable de que sus parejas se molesten y agredan. Por último, el 64\% se rige bajo la creencia de que "el amor es para siempre" y "eres mi alma gemela", ya que piensa que ha encontrado al amor de su vida, motivo por el cual permanece en relaciones conflictivas. 
El centro educativo en el que llevamos a cabo nuestro estudio se encuentra en la ranchería Medellín y Pigua, municipio de Centro, Tabasco, México, que se ubica a treinta minutos del centro de la ciudad. Tiene aproximadamente dos mil habitantes, y es catalogada como una localidad con un grado alto de marginación. Para los jóvenes de nuestro estudio, el pertenecer a esta zona rural representa una desventaja social, por ser un área de asentamiento irregular con carencias de servicios públicos. Sus habitantes tienen pocas oportunidades de trabajo dentro de la comunidad, además de que prevalece un rezago educativo en la población.

Los modelos parentales ante los que han sido expuestos son un caldo de cultivo para que esta etapa se torne complicada, pues los jóvenes han crecido en familias disfuncionales que distan mucho de ser simétricas; en la mayoría de los casos, los jóvenes y niños de esta comunidad viven con sus abuelos o con algún otro familiar, y los que tienen la oportunidad de permanecer con sus padres, crecen observando problemas de violencia intrafamiliar y alcoholismo.

Los usos y las costumbres de la comunidad ponen de manifiesto que temas como sexualidad y noviazgo no son abordados en familia; existen muchos tabús al respecto. Aunado a ello, en el contexto educativo se presentan algunos problemas colaterales, como deserción escolar y embarazo adolescente, problemáticas que mantienen un vínculo con el establecimiento de relaciones amorosas.

A continuación, compartimos los resultados y retomamos el enfoque metodológico de Latorre (2003): describimos los momentos de la intervención, organizados en planificación, acción, observación y reflexión.

\section{RESULTADOS}

\section{Planificación}

La observación participante duró dos meses. Posteriormente, organizamos grupos focales para identificar la percepción, significados y creencias respecto al amor romántico.

Tabla 1. Diagnóstico

\begin{tabular}{|c|c|c|}
\hline $\begin{array}{c}\text { Elemento } \\
\text { constitutivo }\end{array}$ & Significado & Testimonio \\
\hline $\begin{array}{c}\text { Idealizan las rela- } \\
\text { ciones amorosas } \\
\text { Visualizan el amor de } \\
\text { pareja como el único medio } \\
\text { posible para alcanzar la } \\
\text { felicidad y desestiman los } \\
\text { aspectos negativos }\end{array}$ & $\begin{array}{c}\text { "Quizá ideal no en todo, pero que tú sí sientes que es } \\
\text { ideal para ti y quieres hacer cualquier cosa para que } \\
\text { siempre estén juntos" (participante 4) }\end{array}$ & $\begin{array}{c}\text { "Yo seré realmente feliz cuando encuentre a esa perso- } \\
\text { na con quien comparta todo" (participante 9) }\end{array}$ \\
\hline $\begin{array}{c}\text { No identifican sig- } \\
\text { nos de violencia }\end{array}$ & $\begin{array}{c}\text { Desconocen los indicadores } \\
\text { de violencia y distorsionan } \\
\text { la percepción que se tiene } \\
\text { de esta }\end{array}$ & $\begin{array}{c}\text { "Es que depende de cada pareja, a algunos les gusta que } \\
\text { les demuestren que sílos quieren y saber que su pareja } \\
\text { siente celos de algún amigo, etcétera" (participante 3) }\end{array}$ \\
\hline $\begin{array}{c}\text { Justifican la vio- } \\
\text { lencia como actos } \\
\text { de amor }\end{array}$ & $\begin{array}{c}\text { El aprendizaje que adquie- } \\
\text { ren del amor romántico lle- } \\
\text { va consigo una connotación } \\
\text { de sufrimiento-amor }\end{array}$ & $\begin{array}{c}\text { "Creo nadie quiere estar con una persona que te mal- } \\
\text { trata } \text { [...], pero, pues, yo diría que solo en algunos casos } \\
\text { donde los dos se aman y luchan a pesar de la situación } \\
\text { que están sobrellevando" (participante 12) }\end{array}$ \\
\hline
\end{tabular}




\begin{tabular}{|c|c|c|}
\hline $\begin{array}{l}\text { Minimizan la vio- } \\
\text { lencia psicológica }\end{array}$ & $\begin{array}{l}\text { No reconocen las impli- } \\
\text { caciones y alcances de la } \\
\text { violencia psicológica, la } \\
\text { vuelven invisible y per- } \\
\text { miten que esta escale y se } \\
\text { experimente violencia física }\end{array}$ & $\begin{array}{l}\text { "Es que hay como diferentes niveles, ¿no? Pues no es lo } \\
\text { mismo un insulto que un empujón. Porque es normal } \\
\text { que el enojo te haga decir cosas que no piensas" (parti- } \\
\text { cipante 5) }\end{array}$ \\
\hline $\begin{array}{l}\text { Perciben la } \\
\text { violencia como } \\
\text { algo normal en } \\
\text { las relaciones de } \\
\text { noviazgo }\end{array}$ & $\begin{array}{l}\text { El amor romántico consi- } \\
\text { dera la díada sufrimiento- } \\
\text { amor, de manera que } \\
\text { consideran normal que en } \\
\text { las relaciones de pareja ocu- } \\
\text { rran episodios aversivos }\end{array}$ & $\begin{array}{l}\text { "Hay parejas donde afuera pueden estar peleando feo, y } \\
\text { adentro son felices. Y hay personas que pueden pensar } \\
\text { que no se quieren, que la maltrata, pero están también } \\
\text { equivocados porque también no conocen los motivos" } \\
\text { (participante 2) }\end{array}$ \\
\hline
\end{tabular}

Lo anterior dio pauta para conocer las necesidades latentes. A partir de los hallazgos encontrados en los grupos focales, formulamos una propuesta de trabajo que integró los requerimientos específicos de los adolescentes respecto al tema. En la tabla 2 presentamos las categorías con base en las cuales se dirigió la investigación.

Tabla 2. Categorías de análisis

\begin{tabular}{|c|c|c|}
\hline Categorías & Dimensión & Temas propuestos \\
\hline \multirow{2}{*}{ Amor romántico } & $\begin{array}{c}\text { Significado } \\
\text { Creencias } \\
\text { Demostración }\end{array}$ & $\begin{array}{c}\text { Idealización de la pareja } \\
\text { La prueba de amor } \\
\text { Celos }\end{array}$ \\
& $\begin{array}{c}\text { La pareja ideal } \\
\text { El amor lo puede todo }\end{array}$ \\
\hline Violencia & $\begin{array}{c}\text { Tipos de violencia } \\
\text { Señales de violencia }\end{array}$ \\
\hline Noviazgo & Dinámica de la relación \\
Cortejo & Límites en el noviazgo \\
\hline
\end{tabular}

\section{Acción}

El objetivo de esta intervención consiste en modificar las creencias del amor romántico que tienen los adolescentes a fin de prevenir la violencia en las relaciones amorosas. La razón de ello responde a las necesidades específicas de los adolescentes, necesidades no-académicas, pero sí formativas para el desarrollo personal. Además, la oportunidad de los adolescentes de ser parte de este proyecto en esta etapa de sus vidas representa la posibilidad de que generen conciencia sobre el tipo de relaciones que establecerán en la edad adulta.

En esta fase recurrimos a la técnica de taller participativo. La intervención estuvo compuesta por seis sesiones, con los siguientes contenidos: idealización de pareja, la prueba de amor, los celos, la pareja ideal, el amor lo puede todo e identificación de la violencia en el noviazgo. El contenido de las sesiones responde de manera directa a las necesidades del grupo, sobre estas giran sus inquietudes, problemas y 
experiencias en este momento de sus vidas. Para el desarrollo de las sesiones, utilizamos técnicas participativas, como mesa de debate, roll playing, sociodrama, lluvia de ideas, entre otras.

Cabe mencionar que el contenido de las sesiones no es estático; es un proceso reflexivo que permite trabajar desde los conocimientos y las experiencias de los jóvenes y asumir la importancia de la perspectiva de los sujetos, más que de la mirada del investigador.

\section{Observación}

En esta fase empleamos las técnicas de registro de audio y el diario del investigador para documentar el impacto de la intervención en los adolescentes. La tabla 3 contiene los hallazgos.

Tabla 3. Efectos de la acción en los participantes

\begin{tabular}{|c|c|}
\hline Antes & Después \\
\hline $\begin{array}{l}\text { Consideraban el amor de romántico; no analiza- } \\
\text { ban ni se cuestionaban; solo experimentaban }\end{array}$ & $\begin{array}{l}\text { Lograron cuestionar las actitudes y los pensamientos que } \\
\text { tenían sobre la naturaleza del amor romántico }\end{array}$ \\
\hline $\begin{array}{l}\text { Relacionaban el amor de verdad con la supera- } \\
\text { ción de problemas económicos, malos tratos o } \\
\text { adicciones }\end{array}$ & $\begin{array}{c}\text { Comprendieron que el amor no puede desdibujar todos los } \\
\text { obstáculos }\end{array}$ \\
\hline $\begin{array}{c}\text { Consideraban el amor de pareja la única forma } \\
\text { de ser feliz }\end{array}$ & $\begin{array}{l}\text { Reconocieron que amar es una parte importante para ser } \\
\text { felices, pero no la única }\end{array}$ \\
\hline $\begin{array}{l}\text { Normalizaban el sufrimiento en las relaciones } \\
\text { amorosas }\end{array}$ & $\begin{array}{l}\text { Identificaron que el amor de pareja debe ser motivo de } \\
\text { alegría y bienestar }\end{array}$ \\
\hline $\begin{array}{l}\text { Consideraban que la pareja ideal solo se encuen- } \\
\text { tra una vez y hay que cumplir sus expectativas }\end{array}$ & $\begin{array}{l}\text { Identificaron que esta creencia impide ser realistas y ver } \\
\text { los defectos de la pareja }\end{array}$ \\
\hline $\begin{array}{l}\text { Justificaban los gritos, chantajes y el control } \\
\text { como debilidades de la pareja }\end{array}$ & $\begin{array}{l}\text { Identificaron que estos forman parte de la violencia psico- } \\
\text { lógica }\end{array}$ \\
\hline Relacionaban los celos con expresiones de amor & $\begin{array}{l}\text { Asumieron que los celos son una manifestación de violen- } \\
\text { cia que limita la libertad del otro/a }\end{array}$ \\
\hline No visibilizaban la violencia psicológica & Reconocieron las señales de violencia psicológica \\
\hline $\begin{array}{l}\text { Percibían la violencia como forma de interac- } \\
\text { tuar con la pareja }\end{array}$ & $\begin{array}{l}\text { Identificaron que la violencia no es parte de una relación } \\
\text { de pareja saludable }\end{array}$ \\
\hline
\end{tabular}

\section{Reflexión}

En esta fase hicimos una entrevista grupal que nos permitió valorar y reflexionar sobre los conocimientos, aprendizajes y prospectivas de los adolescentes con relación al trabajo realizado. Presentamos a continuación el discurso de los participantes como principales árbitros de este proceso. 


\section{En cuanto a los aprendizajes percibidos:}

Yo creo que aprender a llevar una relación sana con tu pareja, cuando tengas una pareja (sujeto 9).

Mmm [...] A distinguir qué es amor y qué violencia (sujeto 12).

Bueno, son muy interesantes porque aprendí con ellos sobre las cuestiones de una relación de pareja, quizá como lo bueno y lo malo [...] y cómo se debe de llevar una relación sin violencia y sin celos (sujeto 9).

Los adolescentes consideran que el trabajo desarrollado ha sido favorable para la adquisición de conocimientos que no tenían; en ese sentido, se rescata la participación como elemento en el aprendizaje significativo, lo cual se visualiza en los siguientes discursos:

Pues yo he aprendido a desenvolverme mejor en mi relación, comunicarme mejor, hablar de ciertos temas que a veces se omiten y así (sujeto 1 ).

Pues yo he aprendido mucho, creo que también a distinguir entre lo bueno y lo malo, por decirlo así. Recuerdo en la sesión donde teníamos que abordar ciertas creencias del amor romántico [...], recuerdo que la mayoría jalábamos para un lugar y luego pensábamos [...] y nos dábamos cuenta de por qué elegimos esa, y entonces te quedabas pensando... (sujeto 5).

Al respecto de los cambios a nivel personal, mencionan:

Pues creo que ser más consciente de los celos, de lo que puede llegar a suceder o provocar en la violencia en el noviazgo (sujeto 10).

Pues he cambiado de cierta forma mis pensamientos, pues lo que pensaba antes, ya no lo pienso ahora (sujeto 2).

Creo que me ayudan para no cometer errores y no confundir el amor con la violencia, para darme cuenta [...], para prevenirme antes de... (sujeto 3 ).

Los conocimientos adquiridos permitieron generar conciencia crítica sobre el modelo del amor romántico y establecer pautas para prevenir la violencia en futuras relaciones de pareja.

Sobre cambios a nivel grupal, expresan:

Pues aprendí a, por ejemplo, temas que no conocía, a las opiniones de los demás, y también a tomar como lo positivo de los demás, o sea, tal vez aunque no estuviéramos como que no todos pensáramos lo mismo, pero llegáramos a una conclusión, entonces, aprendí todo eso (sujeto 3).

Pues unos somos más que otros [abiertos], creo que ya tratamos de respetar el punto de vista de cada uno, de acuerdo al tema, sin querer imponer algo (sujeto 2).

El trabajo grupal resultó ser un parámetro importante para la adquisición y apropiación de conocimientos, pues los adolescentes desarrollaron confianza para expresarse, diálogo, análisis, reflexión, crítica y confrontación de perspectivas.

Algunas prospectivas reflejan lo siguiente:

Pues de dar nuestra experiencia y de los conocimientos que ya tenemos. Ya nos sentimos capaces de hablar sobre esto, porque ya, o sea, más o menos ya los conocemos (sujeto 2). 
Pues sé que no somos expertos, pero pues creo que algo de lo que aprendimos podemos compartirlo con otros adolescentes (sujeto 7).

Además, creo que es necesario [compartir] porque así como nosotros no sabíamos algunos temas, me imagino que los demás igual, o sea, que les hace falta (sujeto 6).

Los participantes consideran que después de esta experiencia pueden contribuir a prevenir la violencia en las relaciones de noviazgo; aseguran sentirse capaces de "replicar" sus conocimientos con otros adolescentes, al ser agentes de cambio.

\section{ConClusión}

El desarrollo de esta experiencia visibilizó cambios importantes en la concepción de los adolescentes, ya que el proceso permitió reflexionar sobre las implicaciones del modelo hegemónico del amor romántico. Explorar los conocimientos y significados del amor y la violencia contribuyó a que el grupo deconstruyera y transformara sus aprendizajes, al socializarlos en un contexto donde se desdibuja la posibilidad de cambiar paradigmas. Esto se debe a que los jóvenes han crecido observando cómo las expresiones de violencia psicológica y física forman parte de la vida en pareja, sin espacios para replantear, reflexionar y cuestionar dichos comportamientos. Trabajar desde este enfoque hizo posible que los adolescentes cambiaran su percepción sobre las relaciones afectivas, pues las consideraban como la única forma de lograr desarrollo personal, una idea arraigada en esta comunidad.

La intervención participativa en este contexto representó la oportunidad de sumar esfuerzos hacia problemáticas asociadas como embarazos en adolescentes, que está estrechamente ligada a la creencia de la prueba de amor, y la deserción escolar, ya que los adolescentes priorizan la vida en pareja, pues en esta comunidad se asigna un valor al establecimiento de relaciones formales.

A manera de conclusión, la intervención participativa logró que los adolescentes se apropiaran de los contenidos generados desde una postura crítica, más que de la tradicional entrega de contenidos que se hace usualmente, en la que ellos no forman parte de la construcción del conocimiento; por ende, suelen no reproducir contenidos de los que no se apropian. Este abordaje les permitió crear sus propios conocimientos a partir de sus experiencias y reflexiones, lo que puede tener un mayor impacto a futuro en sus relaciones de pareja.

\section{REFERENCIAS BIBILIOGRÁFICAS}

Balcázar, F. E. (2003). Investigación acción participativa (iap): aspectos conceptuales y dificultades de implementación. Fundamentos en Humanidades, vol. 4, num. 7-8, pp. 59-77. Recuperado de http://fundamentos.unsl.edu.ar/pdf/ revista-7-8.pdf

Bonilla- Algovia, E. y Rivas-Rivero, R. (2018). Mitos del amor romántico en una muestra de futuras y futuros docentes. Revista de Estudios e Investigación en Psicología de la Educación, vol. 5, núm 2, pp. 113-117. Recuperado de https:// revistas.udc.es/index.php/reipe/article/view/reipe.2018.5.2.3624/pdf 
Borrajo, E., Gámez, G. \& Calvete, Z. (2015). Justification beliefs of violence myths about love and cyber dating abuse. Psicothema, vol. 27, núm. 4, pp. 327-333. Recuperado de http://www.psicothema.com/pdf/4270.pdf

Bosch, E., Ferrer, V. y Alzamora, A. (2006). El laberinto patriarcal. Reflexiones teóricas- prácticas sobre la violencia contra las mujeres. España: Anthropos.

Bosch, E., Ferrer, V. y Gili, M. (1999). La historia de la misoginia. Barcelona: Anthropos.

Caro, C. (2008). Un amor a tu medida. Estereotipos y violencia en las relaciones amorosas. Revista de Estudios de Juventud, núm. 83, pp. 213-229. Recuperado de http://www.injuve.es/sites/default/files/RJ83-14.pdf

Caro, C. y Monreal, M. (2017). Creencias del amor romántico y violencia de género. International Journal of Developmental and Educational Psychology, vol. 2, núm.1, pp. 47-56. Recuperado de http://www.infad.eu/RevistaINFAD/ OJS/index.php/IJODAEP/article/view/917/796

Cubells, S. y Calsamiglia, J. (2015). El repertorio del amor romántico y las condiciones de posibilidad para la violencia machista. Universitas Psychologica, vol.14, núm 5, pp. 1681-1694. Recuperado de https://revistas.javeriana.edu. co/index.php/revPsycho/article/view/10734

Esteban, M. y Távora, A. (2008). El amor romantico y la subordinacion social de las mujeres: revisiones y propuestas. Anuario de Psicología, vol. 39, núm. 1, pp. 59-73. Recuperado de https://revistes.ub.edu/index.php/Anuario-psicologia/article/view/8394/10359

Ferrer, V. y Bosch, E. (2013) Del amor romántico a la violencia de género. Para una coeducación emocional en la agenda educativa. Revista de Currículum y Formación del Profesorado, vol. 17, núm. 1, pp. 106-122. Recuperado de https://recyt.fecyt.es/index.php/profesorado/article/view/41570

Flores, V. M. (2019). Mecanismos en la construcción del amor romántico. Revista de Estudios de Género La Ventana, vol. 6. núm. 50, pp. 282-305. Recuperado de http://revistalaventana.cucsh.udg.mx/index.php/LV/article/ view/7074/6166

Galicia, I., Sánchez, A. y Robles, F. (2013). Relación entre estilos de amor y violencia en adolescentes. Psicología desde el Caribe, vol. 30, núm. 2, pp. 211-235. Recuperado de http://rcientificas.uninorte.edu.co/index.php/psicologia/article/viewFile/4854/3351

García, J., Hernández, C. I. y Monter, N. S. (2019). Amor romántico entre estudiantes universitarios (hombres y mujeres), una mirada desde la perspectiva de género. Revista de Estudios de Género La Ventana, vol. 6, núm. 49, pp. 218247. Recuperado de http://revistalaventana.cucsh.udg.mx/index.php/LV/ article/view/6996/6111

Guzmán, G. y Bolio, M. (2010). Construyendo la herramienta de perspectiva de género: cómo portar lentes nuevos. México: Universidad Iberoamericana.

Instituto Estatal de las Mujeres (IEM Tabasco, 2018). Diagnóstico de violencia en el noviazgo en jóvenes de 15 a 18 años del estado de Tabasco. Recuperado de https://transparencia.tabasco.gob.mx/media/estrados/6869.pdf

Instituto Nacional de Estadística y Geografía (INEGI, 2016). Encuesta Nacional sobre la Dinámica de las Relaciones en los Hogares (ENDIREH). Principales resultados. Recuperado de https://www.inegi.org.mx/contenidos/programas/ endireh/2016/doc/endireh2016_presentacion_ejecutiva.pdf 
Lagarde, M. (2001). Los cautiverios de las mujeres: madresposas, monjas, putas, presas y locas. México: Universidad Nacional Autónoma de México.

Larrañaga, E., Yubero, S. y Yubero, M. (2012). Influencia del género y del sexo en las actitudes sexuales de estudiantes universitarios españoles. Summa Psicológica UST, vol. 9, núm. 2, pp. 5-13. Recuperado de https://summapsicologica. cl/index.php/summa/article/view/89

Latorre, A. (2003). La investigación-acción. Conocer para cambiar la práctica educativa. Chile: Graó.

Marroquí, M. y Cervera, P. (2014). Interiorización de falsos mitos del amor romántico. Reidocrea, vol. 3, pp. 142-146. Recuperado de https://digibug.ugr. es/bitstream/handle/10481/32269/ReiDoCrea-Vol.3-Art.20-Marroqui-Cervera.pdf? sequence $=1 \&$ isAllowed $=y$

Organización Mundial de la Salud (OMS, 2017). Violencia contra la mujer. Datos y cifras. Recuperado de https://www.who.int/es/news-room/fact-sheets/ detail/violence-against-women

Pedroza, F. R. (2011). La investigación-acción en la práctica educativa reflexiva. México: CICE e-ikon digital. Recuperado de https://issuu.com/renepedroza/ docs/libro.3ra.edicio_n_iape

Rodríguez, T. (2017). El amor y la pareja. Nuevas rutas en las representaciones y prácticas juveniles. México: Universidad de Guadalajara.

Rodríguez-Castro, Y., Lameiras-Fernández, M. y Carrera-Fernández, M. V. (2015). Amor y sexismo: una peligrosa relación en los y las adolescentes gallegos/as. Revista de Estudios e Investigación en Psicología y Educación, vol. extr., núm 2, pp. 55-59. Recuperado de https://revistas.udc.es/index.php/reipe/article/ view/234/pdf_13

Valenzuela, J. (2004). Género y repertorios identitarios. En A. de la Torre, R. Ojeda y C. Maya. Construcciones de género en sociedades con violencia: un enfoque multidisciplinario (pp. 51-68). México: Porrúa.

Yela, C. (2002). El amor desde la psicología social. Ni tan libres, ni tan racionales. Madrid: Ediciones Pirámide. 